\title{
Analysis of multidimensional spectra: estimating the continuum
}

\author{
H.C. Griffin and M.G. Chemaly \\ Department of Chemistry, University of Michigan, Ann Arbor, MI 48109-1055, USA
}

\begin{abstract}
We have developed a two-dimensional fitting program which extracts coincidence intensities and their uncertainties from a $\gamma-\gamma$ coincidence matrix. The data are fitted by peaks and ridges on a two-dimensional continuum. The continuum is described as the product of components of a presumed Compton distribution for the set of detectors contributing to each axis of the continuum. Peaks and ridges are based on simple Gaussians
\end{abstract}

\section{Introduction}

Experımental investigations of excited states of nuclei populated in heavy-ion reactions involve arrays of detectors, generally including different types of gamma-ray detectors (scintillators as well as semiconductors) and perhaps particle detectors. Although each event includes at least two high-resolution gamma-ray detectors, the most common method of analysis involves setting gates on all parameters but one and analyzing that parameter for peaks. This projection method - as opposed to fitting in many-dimensional space - was necessary when computer memories were relatively small (less than a megabyte available to a single user).

Two-dimensional spectra (correlations between two detectors) require storage of a few tens of megabytes. Current workstations either have enough memory to hold the entire array or can access the entire array from disk with a small amount of paging. (Some laboratories are emphasizing events which involve three high-resolution detectors; these cannot be histogrammed, and in any event could not be decomposed directly.)

Emelianov et al. [1] developed programs for direct decomposition of coincidence spectra of capture gamma rays in odd-odd nuclei. They argued that the projection method was ineffective for their spectra, in which the average distance between gamma rays was comparable to energy resolution (FWHM). The method is based on a priori information of energies and intensities of expected gamma rays. The full matrix is divided into submatrices small enough to be handled in avalable computers. The submatrix is compared with a model composed of a continuum, ridges and (two-dimensional) peaks. The model is adjusted to give the best fit.

Vanın and Aiche [2] argued that two-dimensional fits involve an excessive number of degrees of freedom and returned to one-dimensional fits. Their single-parameter spectra were constructed so as to eliminate contribu- toons from the continum and neighboring peaks (based on empirical line shapes and a prori energies of gamma rays). Particular attention was given to the propagation of statistıcal errors.

We have reinvestigated direct decomposition of the coincidence matrix, and tested the analysis with data from an array of Ge detectors observing a heavy-ion reaction [3]. These data consisted of a 4096 channel by 4096 channel matrix obtained from eight $\mathrm{Ge}$ and $\mathrm{Ge}(\mathrm{Li})$ detectors gated by high $\gamma$-ray multiplicity. The data were selected because more than half of the events arise from unsuppressed detectors, implying that the continuum and ridges are important features of the data.

\section{Method of analysis}

Our initial attempts to fit $2 \mathrm{D}$ spectra were similar to that of Emelianov et al. [1], namely, we described the contınuum as a tılted plane in each of the fitting regions (generally the rectangle defined by adjacent regions relatively free of peaks in the total projection spectrum) We anticipated that a fairly small number of parameters (many fewer than the number of channels) could be used and that the fits could be checked visually.

A two-dimensional histogram of a relatively high count region of the matrix is given in fig. 1 . It is clear that statistical fluctuations obscure all but the most obvious features. Additional difficulties concerned how those regions should join and whether we should accept backgrounds with clear discontınuities between fittıng regions. We concluded that a more realistic continuum function was needed.

One-dimensional analyses depend on substantial information about the context (smoothness, continuity, correlations, etc.) of the data, and similar information is needed for two-dimensional analyses. As Emelianov et al. [1] point out, the primary features of the spectrum 


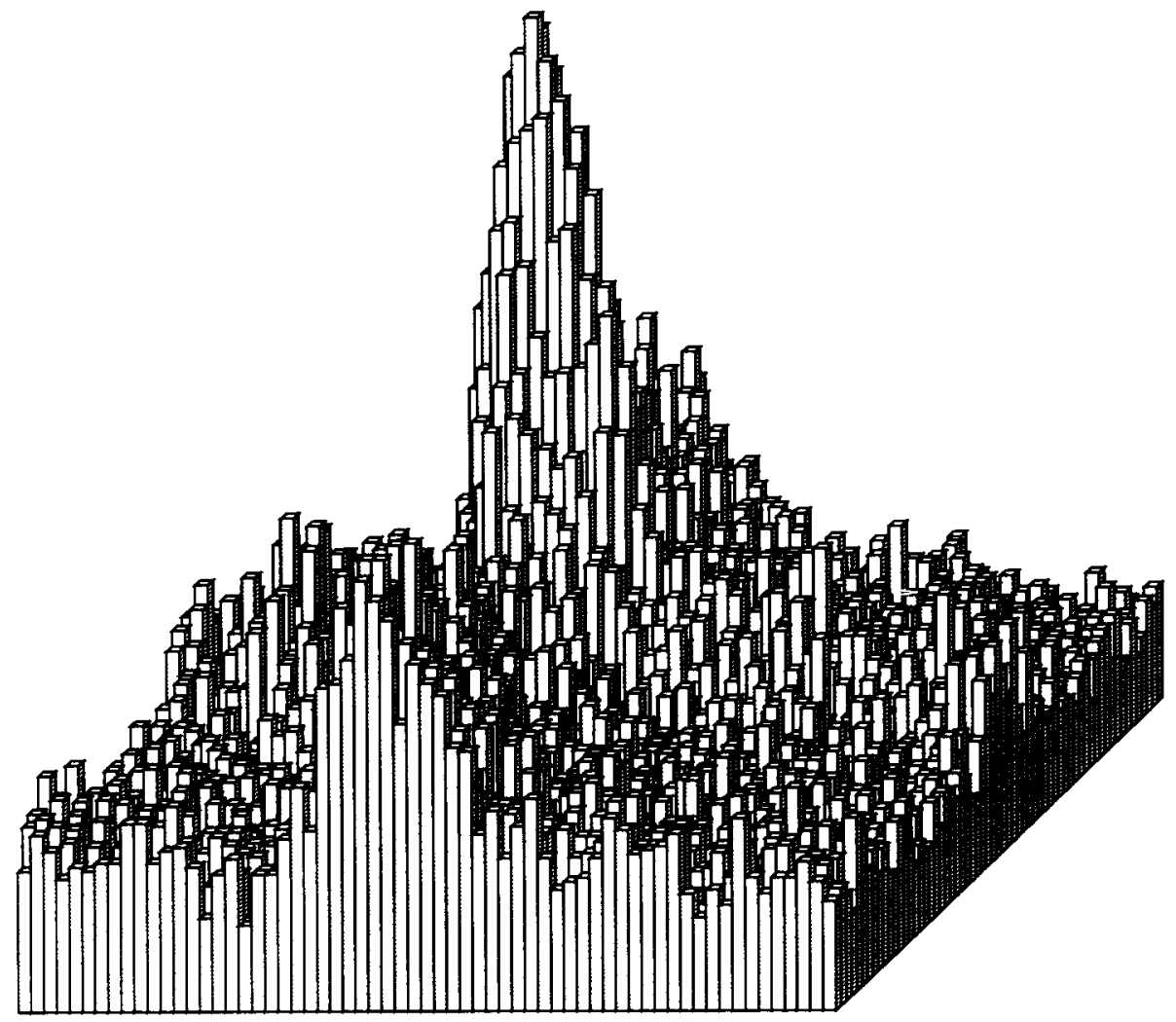

Fig. 1. Part of a $\gamma-\gamma$ coincidence matnx from the ${ }^{122} \mathrm{Sn}\left({ }^{45} \mathrm{Sc}, 4 n\right){ }^{163} \mathrm{Lu}$ reaction. This region has one of the highest counts per channel of the $4096 \times 4096$ matrix. Conversion gain is $0.3 \mathrm{keV}$ per channel. The highest peak is a concidence between 198- and 293-keV $\gamma$-rays.

are a continuum (from a continuum, primarily from Compton interactions, in each detector), ridges (from coincidences between a peak and a continuum) and peaks (from peaks in each detector). If these are the correct interpretations of the origins of the features, there should be at most one continuum for each detector which describes most of the data in the two-dimensional spectrum. And the continuum should be similar to the background used in one-dimensional analyses.

\subsection{The continuum}

The continuum is described by

$A(i, j)=H(i) \times H(j)$,

where $H(l)$ is the height of the model Compton distribution in channel $t$ and $A(i, J)$ is the area of the 2D continuum in channel $(l, j)$. The total projection shows 24 regions which are relatively free of peaks and spaced so as to define the continuum. This means that the 576 intersections of 24 regions on each axis should be good approximations to the 2D continuum. Starting with an initial estimate

$C(l)=A(l, i)$,

we determined the best values for the average of the function $H(t)$ in each of the 24 peak-free regions by weighted least-squares fitting to the 576 intersections. These values were found to describe the intersections very well. The distribution of differences is shown in fig. 2. Here the average number of counts per channel in the region is compared with the calculated value in terms of standard deviations, where the error is computed from the total number of counts in the region. The distribution is slightly broader than simple statistics would predict; it is likely that some of the "background" regions contain peaks or tails of peaks. Fig. 3 shows the 


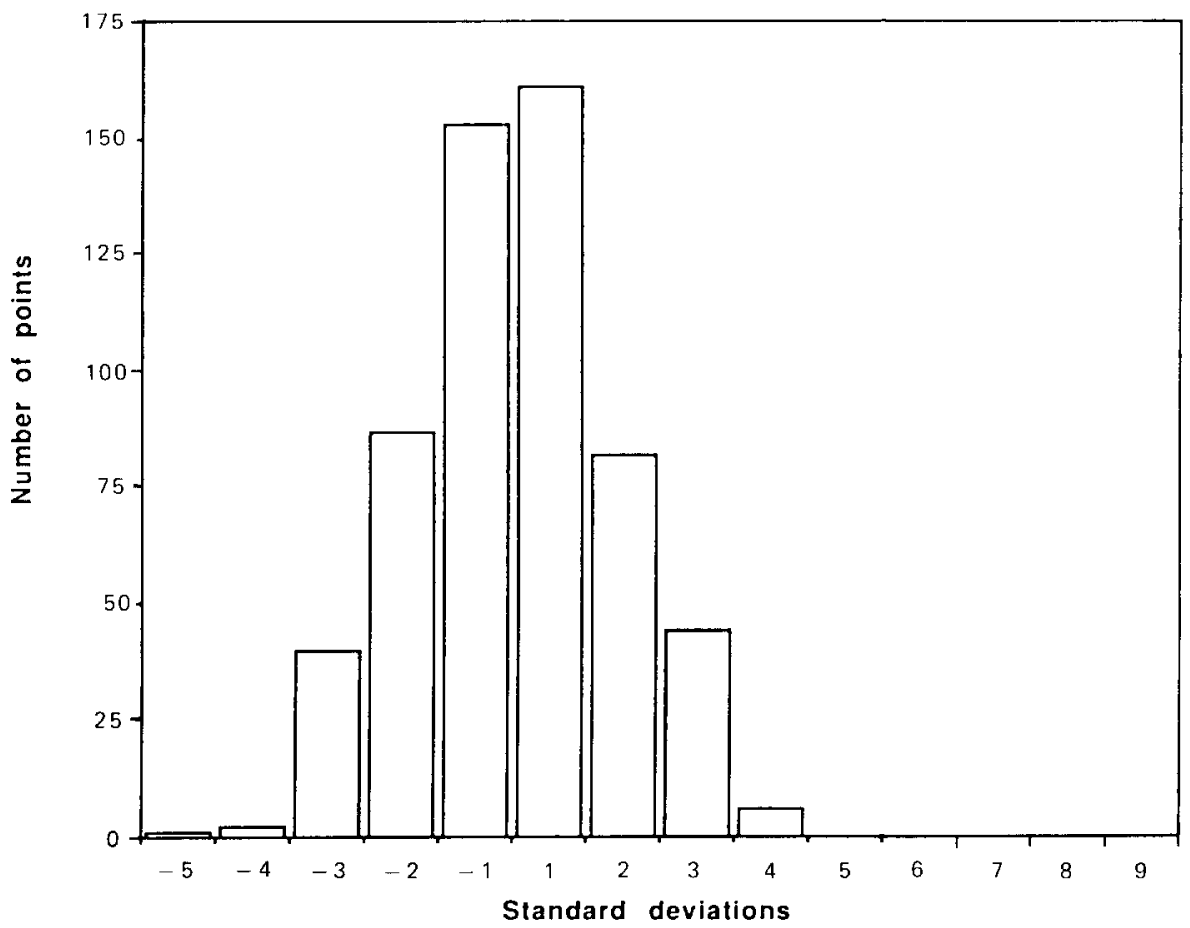

Fig. 2. Distribution of background regions. The abscisca is data minus model in terms of standard deviations based on total data counts. The ordinate gives the number (out of 574) of regions.

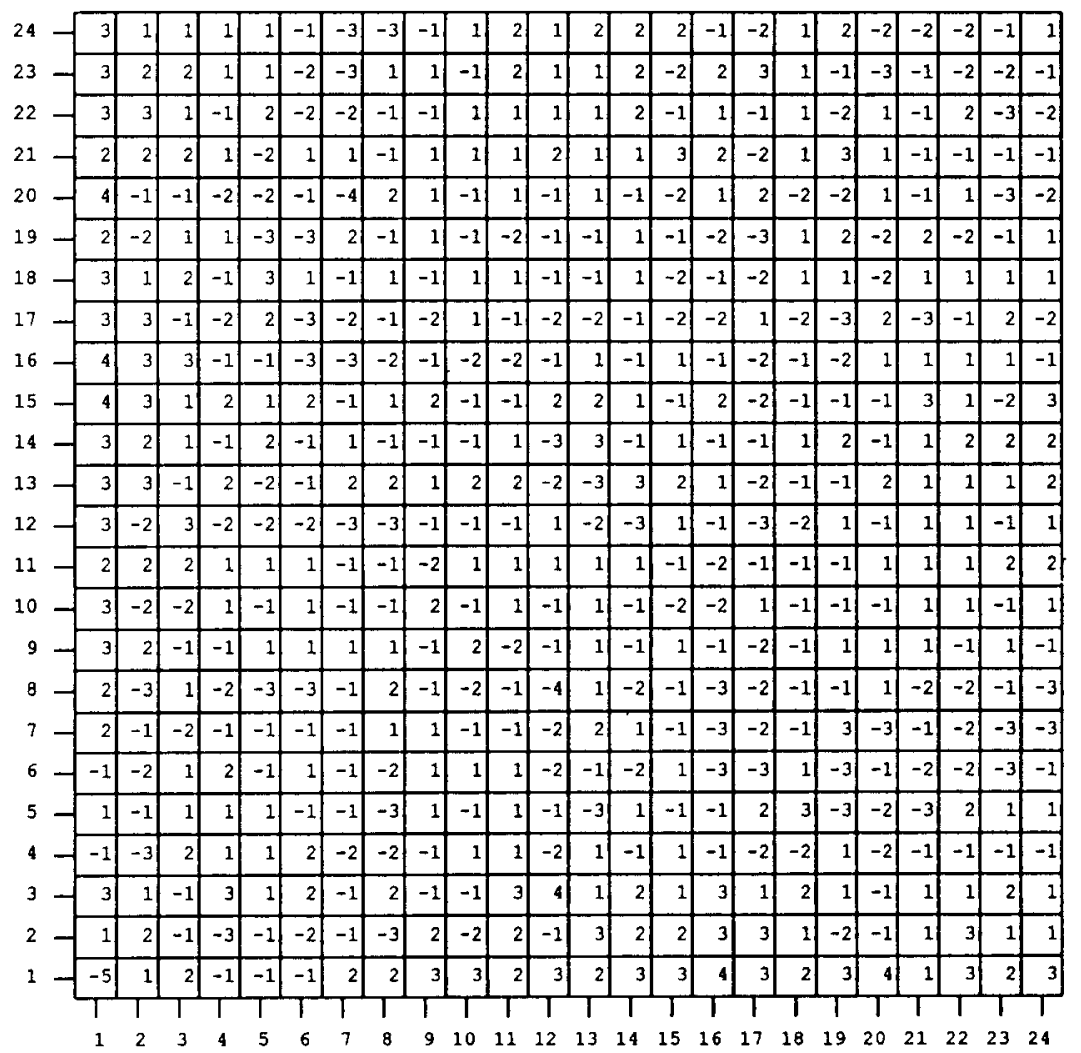

Fig. 3. Map of background regions. Numbers along the axes identify the background regions. Entries in the array are the data minus model in terms of standard deviations. 
map of these differences. There are some "ridges" and "valleys" in the map, implying that some of the components of the "Compton" distribution are too high or too low. For example, many of the values corresponding to the 15th component have negative values for (observed - calculated) values. The first and second components are near the $\mathrm{K} \mathrm{X}$-rays of $\mathrm{Lu}$, where the data are considerably higher than the fits due to the very low value on the diagonal. Aside from these trends, which account for most of the extra width of the distribution (fig. 2), the agreement is very good.

\subsection{The ridges}

The projection of the matrix shows at least 205 distinct peaks. The same 24 regions described above were used to determine the height of the ridge associated with each of these peaks. Thus $205 \times 24$ heights were determined. The axis normal to the ridges was described as Gaussian functions of fixed position and width (from decomposition of the matrix projection). For each of the 24 regions, the 205 heights were successively determined (by least squares) and the process repeated until suitable convergence.

The 24 components for each peak were compared to the function $H(i)$. There were gross similarities, but the ridges tended to be higher at high energies, and there were significant differences among ridges.

\subsection{The peaks}

The peaks were defined by a height for each of the $205 \times 205$ intersections of the peaks resolved in the projection; positions and widths as well as properties of ridges and the continuum were fixed. Again, the process was repeated until convergence.

\subsection{Uncertaintles}

For each of the fitting stages, uncertanties were determined by varying each datum (counts per channel) and recomputing the fits in affected parameters. These calculations showed that the major errors were due to limited counts in peak regions and overlap in peaks.

\section{Conclusions}

Relative contributions of the several model components are compared in fig. 4 , which shows projections near $200 \mathrm{keV}$ (a complex region of overlapping peaks) and $620 \mathrm{keV}$ (the highest energies with moderate intensities). In each part of the figure, the projection of the continuum is the lowest line. Adding the projection of parallel ridges gives the next line. Adding ridges normal to the axis gives the smooth spectrum, which should not exceed the data. The remaining distance to the data
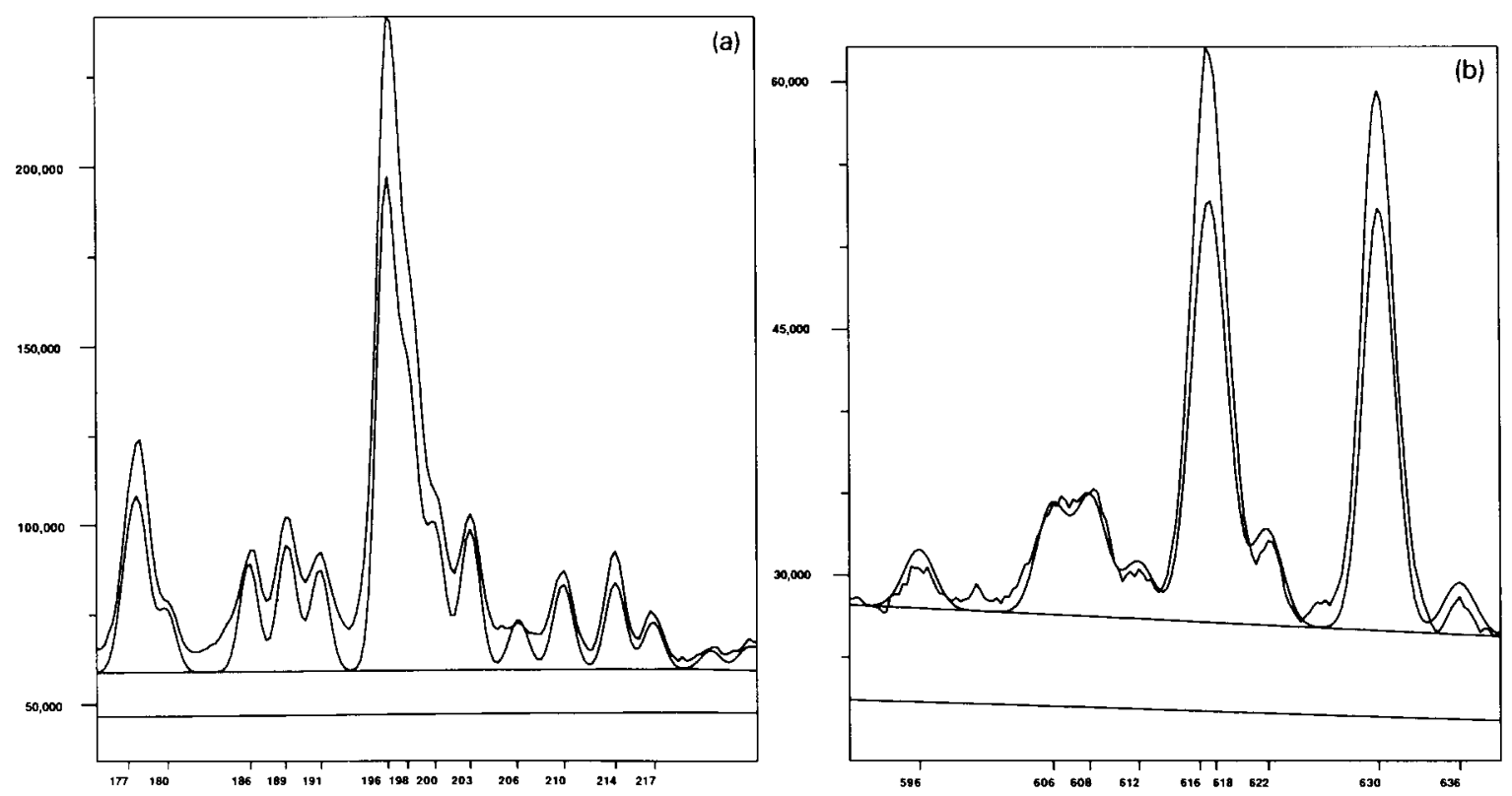

Fig. 4. Comparison of spectral contributions. (a) The projections in the vicinity of the 198-keV group. The top curve is the total projection. The straight line at the bottom ( 46000 counts) is the projection of the calculated continuum. Adding the projection of parallel ndges gives the upper straight line (53000 counts). Adding the projection of ridges perpendicular to the projection axis gives the lower curve. The hash marks and numbers below the abscisca identıfy the energies and positions of $\gamma$-rays. (b) The projections in the vicinity of the $617-\mathrm{keV}$ group. 
projection corresponds to the projection of peaks. It appears that our model is quite adequate for the continuum, but that the ridges need considerable refinement.

\section{References}

[1] B.A. Emelianov, L.P. Kabına, I.A. Kondurov, Y.E. Loginov and P.A. Sushkov, Nucl. Instr. and Meth. 178 (1980) 555
[2] V.R. Vanın and M. Aiche, Nucl. Instr. and Meth. A284 (1989) 452.

[3] K. Honkanen et al., in. Nucleı Off the Line of Stability, eds. R.A. Meyer and D.S. Brenner, ACS Symposium Series 324 (Am. Chem. Soc., Washıngton, DC, 1986) p. 305. 